\title{
Research of D2D Dynamic Power Control Algorithm Based on Interference Restricted Area in Multi-Cell
}

\author{
Janbin Xue ${ }^{1}$, Supan $\mathrm{Wei}^{2}$ and Yiming Chen ${ }^{3}$ \\ ${ }^{1,2,3}$ Institute of Computer and Communication, Lanzhou University of Technology, \\ Lanzhou 730050, GanSu, China (e-mail: weisupan0304@163.com.)
}

\begin{abstract}
In cellular networks, the application of device-to-device (D2D) technology can effectively improve the system spectral efficiency. In order to research the performance of D2D communication in multi-cell cellular system, this paper proposed a dynamic power control algorithm based on interference restricted area to solve the interference problem between D2D communication and cellular communication in multi-cell. First we use Laplace Transform to analyze the interference, then based on the analyze of outage probability and area spectral efficiency, through system-level simulation, research the impact of D2D user equipment density, D2D communication distance and different interference restricted area sizes on outage probability and area spectral efficiency. Simulation results show that interference restricted area can effectively reduce communication outage probability, as well as improve system area spectral efficiency.
\end{abstract}

Keywords: D2D communication; area spectral efficiency; outage probability; interference restricted area

\section{Introduction}

As more and more new mobile multimedia services for mobile users to choose, the demand for high-speed wireless access data rates increasing in communication system, a number of new wireless technologies have emerged. Such as LTE-A (long term evolution advanced), WIMAX (world interoperability for microwave access), can provide highspeed, high-capacity mobile services and also to ensure users Qos (quality of service) [1]. On the other hand, the development of cellular network technology promote the "small cell", "micro cell" and other new technologies, so it is possible to improve network capacity by reducing cell size and control interference effectively. However, all these innovations still rely on centralized network topology, mobile devices need to communicate through an access point or BS (base station). Such centralized network topology is often limited by the carrying capacity of BS and access points, once there is a huge number of communication request, the network congestion may occur. And the BS and the access point may not be complete have the transmission parameter information between devices, thus bring difficulty to obtain optimal network performance.

In order to deal with these problems, the concept of D2D (device-to-device) communication is introductive, it allows point-to-point transmission between mobile devices, share part of the data traffic from BS and access points. D2D communication can improve spectrum efficiency, reduce the transmit power of terminals and solve the problem of spectrum resource scarcity that exist in wireless communication system, etc. [2]. As a key technology of the 5G, D2D communication has the potential to improve the system performance, improve the user experience, and expand the application prospect of cellular communication, so it has received increasing attention recently. In the Europe project of Mobile and Wireless Communications Enablers for the Twenty-Twenty (2020) Information Society (METIS 2020), Device-to-Device Communication, Ultra-Dense 
Network, and Ultra-Reliable Communication were introduced into the project as the key technologies of 5G [3].

Compared with the traditional Peer-to-Peer (P2P) technology, a main advantage of D2D communication based on cellular network is that the interference can be controlled. However, D2D technology in cellular networks is bound to bring extra interference. Considering the multiple hops, unauthorized LTE band (LTE-U), application of high frequency communication features in 5G D2D network, the researches of power control and interference coordination are critical.

This paper from the perspective of further improve the system throughput and reduce communication interference, apply restricted area and dynamic power control algorithm to D2D communication. First, considering the D2D communication interference situation in multi-cell, proposed a kind of power control algorithm to reduce inter-cell interference and improve the communication quality of cell edge users. Then, for the four kinds of interference exist when D2D users reuse cellular uplink spectrum resources, based on the analysis of outage probability and area spectrum efficiency, proposed the idea of the restricted area. By setting the appropriate area exclusion radius for base station, make D2D users which cause interference to base station outside the restricted area, so that alleviate interference that brought by $\mathrm{D} 2 \mathrm{D}$ communication.

The rest of this paper is organized as follows. A review of the related work and motivation of this work are presented in Section 2. In Section 3, we present the system model, followed by problem description in Section 4. In Section 5, four type interferences and their Laplace Transform are investigated. The outage probability and area spectral efficiency analysis are given in Section 6. Numerical results and discussion are addressed in Section 7. Finally, conclusions are drawn in Section 8.

\section{Related Work and Motivation}

Although D2D communication is direct communication from terminal to terminal, the communication process is still under control of the base station. In addition to allocate spectrum resource, base station establish the cellular link and D2D sessions by controlling the transmission power of cellular users and D2D users to improve system performance, reduce energy consumption and control inter-link interference effectively.

There are many articles discuss the interference avoidance and coordination from the aspects of power control. In [4], Doppler et al. presented a power optimization scheme based on a single cell model, giving cellular users priority by ensuring their minimum transmission rate, this method increase the total rate of system. Fodor $\mathrm{G}$ et al. [5] proposed a distributed power control algorithm, calculate the SINR optimization target of web users, and assign transmission power to minimize power consumption of system. The authors of [6] considered two kinds of power control algorithms: Centralized Power Control Algorithm and Distributed On-Off Power Control Algorithm. The purpose of centralized power control algorithm is through controlling the transmit power of D2D link to ensure the communication quality of cellular users', at the same time guarantee the number of D2D links reach to maximize. Considering the centralized power control algorithm need base station to get all the channel state information, it will cause a lot of signaling overhead, so Distributed On-Off Power Control Algorithm is proposed in this paper, the main idea of this algorithm is D2D transmitters according to the status of direct link and the nonnegative threshold $\mathrm{G}_{\min }$ that system set to choose their own maximum transmit power, this algorithm does not consider the interference to other D2D users, do not need to mutual cooperation between D2D links. J. Gu et al. [7] studied under the premise of the known of channel state, by adjusting the D2D's transmit power to make base station and cellular users exclude outside the D2D communication coverage area. So that avoid the interference between cellular users and D2D users as far as possible. Min $\mathrm{H}$ et al. [8] put forward an interference exclusion area control strategy. In this strategy, when 
cellular users (CUES) in this area and make serious interference to D2D communication, in order to ensure the priority of cellular communication, the system automatically switches the D2D communication mode to cellular communication mode, thus avoiding the interference between cellular communication and D2D communication, and also increasing the throughput of the system. In [9], the authors proposed an OFDMA system power optimization program, jointly consider resource allocation and mode selection, effectively reduces the downlink transmission power and the base station power consumption. T. F. Maciel et al. [10] analyzed D2D power control under MIMO cellular system, the authors discuss the transmission power optimization problem under the condition of fixed minimum SINR threshold and non-fixed minimum SINR threshold, the results show that power control under the non-fixed minimum SINR threshold can lead to higher performance gain. In [11], the authors proposed a resource scheduling scheme based on interference situation awareness. In this paper, set at only one cell scene, D2D interference can be reduced through the use of resource allocation and power restriction and other methods, but the most important is to ensure the performance of cellular communication which as the main the business not affected too much. D2D terminal can estimate channel fading conditions by measuring the received interference signal, so to adjust and allocate resources to CUEs and DUEs by base station after feedback. By applying the power optimization setting and the best mode selection method, in comparison with the random resource allocation, the SINR of cellular communication links has been significantly improved. In this scheme, the base station needs to obtain all the link channel state information (CSI), so the cost of system control message is very large.

The interference coordination and power control schemes above are most research the performance of the D2D technology under a single cell. In fact, single cell is an ideal system, in the actual system, the communication between users not only suffer the interference from its own cell, but also suffer the interference from neighbor cells, especially for the edge users of cell. The interference environment in multi-cell and single cell is different, this lead to the conclusion in single cell can not be directly applied to multi-cell. So this paper proposed a multi-cell model, in order to reduce inter-cell interference that cellular users and D2D users suffered, design interference restricted area focus on decreasing the interference that generated by D2D communication.

\subsection{System Model}

\subsection{Communication Model}

Communication model is shown in Figure 1. Here we consider many D2D pairs under multi-cell. One cell includes a base station, a cellular user and many D2D users, D2D users communicate with each other by reusing uplink frequency spectrum resource of cellular user. All users establish the connection, allocate resources under the control of BS. Base station is located in the center of the cell with the density $\lambda_{B}$. The location of cellular transmitters and potential D2D transmitters are modeled as two independent homogeneous PPP $\Phi_{C}=\{c 1, \mathrm{c} 2, \ldots\}$ and $\Phi_{\mathrm{D}}=\{d 1, \mathrm{~d} 2, \ldots\}$ with the density $\lambda_{C}$ and $\lambda_{D}$ respectively, the distance between cellular user and base station, D2D transmitter and receiver is $L_{C}$ and $L_{D}$ respectively.

As shown in Figure 2, BS is located in a protected area $\mathrm{Z}$ which called interference restricted area, its radius is r. D2D users located in this area can't be activated, which are not allowed to use reuse mode to communicate, only the D2D users outside this area can be allowed to use reuse mode to communicate with each other. To reduce interference, we assume that the cellular user is located in this area. All cellular users and D2D users keep communication with BS, and the channel state information (CSI) is known by BS. 


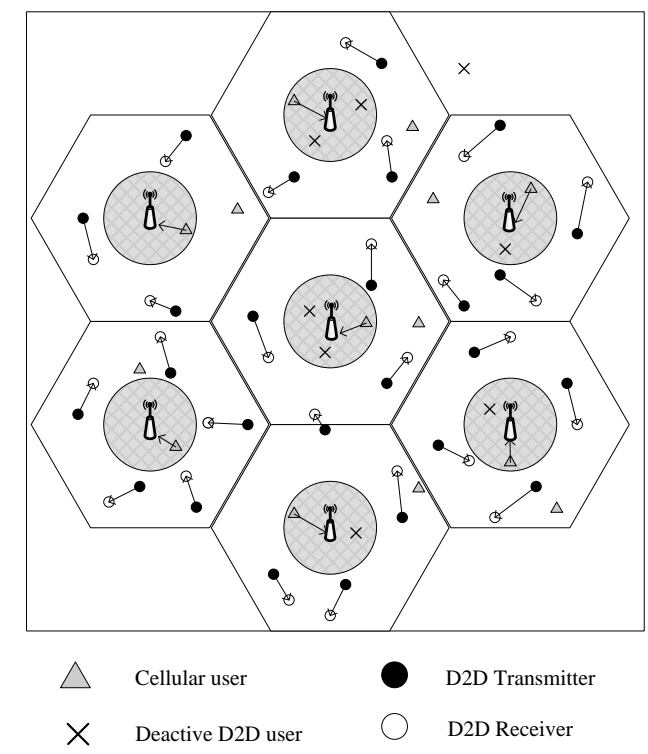

Figure 1. The Scenario of D2D Communications and Cellular Communications Coexistence Under Seven Cells

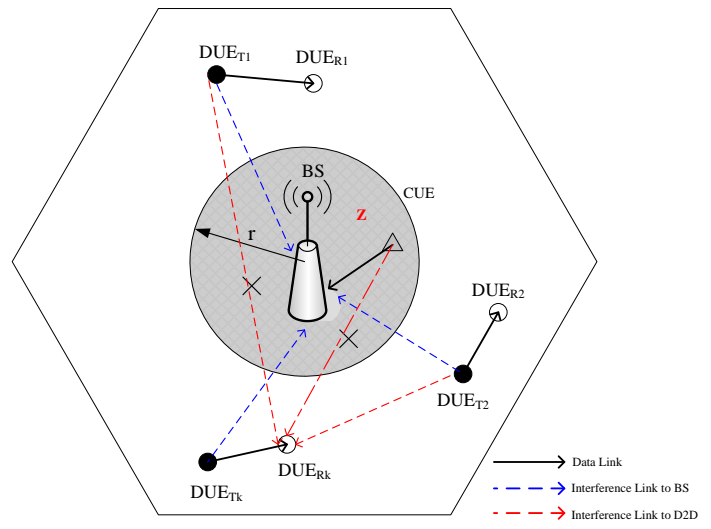

Figure 2. Interference Model in a Single Cell (Consider D2Dk as the Example, all D2D Users Reuse Uplink Frequency Spectrum Resources of CUE to Communicate)

\subsection{Channel Model}

The information between BS and UE exchange through the air channel. In order to establish transmission channel model, we consider the situation that transmitters and the receivers are all single antenna. The physical layer transmit in frame, the data link layer transmit in packet, and a frame may contain one or more data packets. With the following assumptions:

(1) Channel is a non-frequency selective channel, this can be achieved by OFDM technology. Channel parameters remain unchanged at the transmission time of a frame, but it may change between frame and frame, we called this channel block fading channel [12]. Thus, the modulation and coding strategy used for each frame are the same.

(2) D2D receiver can accurately obtain channel state information, and notify the transmitter by no delay and error feedback channel. The transmitter based on feedback information to adjust modulation and coding. 
(3) The receiver determines whether the packet that received is correct based on cyclic redundancy check (CRC). If the packet is correct, give feedback ACK(Acknowledgement) signal to the transmitter, the transmitter start to transmit the next data packet; Otherwise, give feedback NACK signal, the transmitter continue to send the packet until it receives the right or the maximum number of retransmissions.

The length of the packet is $\mathrm{N}_{\mathrm{P}}$, information bit is $\mathrm{L}, \mathrm{CRC}$ bit is $\mathrm{C}$. then we have $\mathrm{N}_{\mathrm{P}}=\mathrm{L}+\mathrm{C}$. ARQ (Automatic Repeat request) is automatic retransmission request, The path loss is inversely proportional to the transmission distance of signal, that is $L(\mathrm{~d}-\mathrm{c})=\|d-c\|^{-\alpha}$. Where $\alpha$ is the path loss exponent, $\mathrm{d}$ and $\mathrm{c}$ represent UE. According to the analysis, establish channel model, as shown in Figure 3.

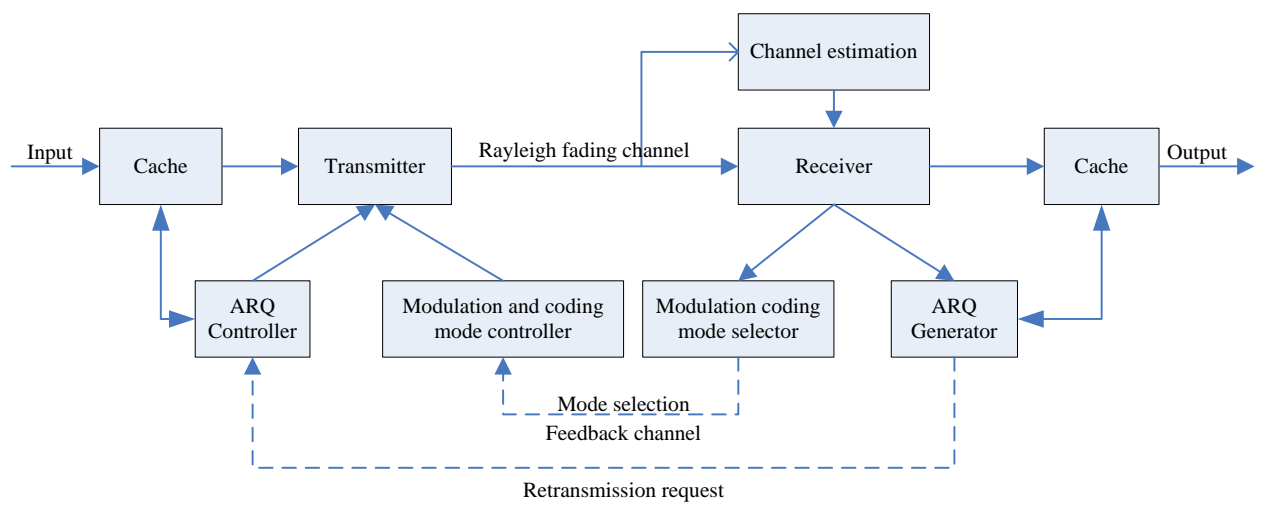

Figure 3. Channel Model

\section{Problem Description}

In the previous section, we have introduced many kinds of power control methods to coordinate interference. In this section, we proposed a new dynamic power control algorithm and the concept of interference restricted area based on the communication model and channel model.

\subsection{Uplink Dynamic Power Control Algorithm}

When calculate the transmit power of communication users, We use open loop dynamic power control algorithm (OFPC) which mentioned in reference [13] to adjust the D2D transmit power. This algorithm point out that transmit power proportional to transmission distance. This allows when D2D transmitter is close to the receiver, D2D transmitter can employ a small transmit power, thereby reducing overall power consumption of D2D communication. According to this algorithm, UEx transmit power can be expressed as:

$$
P_{x}=h x^{\alpha \mu}
$$

Where

$$
h=P_{x \max } / R^{\alpha \mu}
$$

$P_{x \max }<P_{\max }, P_{\max }$ is the maximum transmit power that allowed to mobile terminals, $\mathrm{R}$ is the radius of the base station coverage area and $R=\sqrt{1 /\left(\pi \lambda_{\mathrm{B}}\right)}, \mathrm{x}$ is the distance between the transmitter and the receiver, ${ }^{\mu}$ is Path loss compensation coefficient and $\mu \in[0,1]$. 


\subsection{Interference Restricted Area}

The interference to point y from other users $\Phi_{x}$ can be expressed as:

$$
I_{x y}=\sum_{x \in \Phi_{x}} P_{x} \eta_{x y} L(\mathrm{y}-\mathrm{x})
$$

Where $\Phi_{x}$ is the set of all interferers, $P_{x}$ is the transmit power of interferer $\mathrm{x}, \eta_{x y}$ is Rayleigh fading coefficient, $L(y-x)=\|y-x\|^{-\alpha}$ is path loss from $\mathrm{x}$ to $\mathrm{y}$.

In order to reduce interference between cellular communication and D2D communication, we put forward the thought of interference restricted area, BS is the center of interference restricted area, radius is $\mathrm{r}$, as shown in Figure 2. In this thought, D2D can reuse cellular user's uplink frequency spectrum resources, and only D2D transmitter that outside the restricted area can be activated, all D2D users that located in the restricted area are not allowed to communicate with each other. In order to facilitate analysis, we assume the following:

(1) The radius of interference restricted area is $r$ for all cell, at least one cellular user located in the area.

(2) According to the requirement of system quality of service (QoS), the signal to interference plus noise power ratio (SINR) between cellular link and D2D link threshold is T. The coverage probability of a typical UE can be expressed as:

$$
P=P\{\operatorname{SINR}>T\}
$$

\section{Interference Analysis}

There exist four types of interference in the proposed communication scenario. We take one D2D communication for example: (1) the interference from all CUES to a D2D receiver denoted as $I_{c d} ;(2)$ the interference from other D2D transmitters to a D2D receiver denoted as $I_{d d}$; (3) the interference from all D2D transmitters to BS denoted as $I_{d b} ;$ (4) the interference from other cell CUEs to BS denoted as $I_{c b}$.

In order to analysis facilitate, we use Laplace transform to calculate the interference. First we map the d-dimensional PPP onto $\mathrm{R}^{+}$by letting $\Phi=\left\{r_{i}=\left\|x_{i}\right\|\right\}$ be the distances of the points of a d-dimensional uniform PPP of intensity $\lambda^{\prime}(r)=\lambda c_{d} d^{d-1}$, where $c_{d}=|b(0,1)|$ is the volume of the d-dimensional unit ball. We can identify the path loss law $l(\mathrm{r})=\eta_{\mathrm{r}} \mathrm{r}^{-\alpha}$. Then we would like to calculate the Laplace transform of the interference.

$$
L_{I}(\mathrm{~s})=\mathrm{E}\left[e^{-s l}\right]=E\left[\exp \left(-\mathrm{s} \sum_{r \in \Phi} \eta_{r} r^{-\alpha}\right)\right]
$$

The expectation is to be taken over both the point process and the fading. Due to the independence of the fading

$$
L_{I}(\mathrm{~s})=E_{\Phi}\left[\prod_{r \in \Phi} E_{\eta}\left[\exp \left(-\mathrm{s} \eta_{r} r^{-\alpha}\right)\right]\right]
$$

This is a probability generating functional with $v(\mathrm{r})=E \exp \left(-s \eta_{\mathrm{r}} \mathrm{r}^{-\alpha}\right)$, so we have

$$
L_{I}(\mathrm{~s})=\exp \left\{-E_{\eta}\left(\int_{0}^{\infty}\left(1-\exp \left(-s \eta r^{-\alpha}\right)\right) \lambda^{\prime}(\mathrm{r}) \mathrm{dr}\right)\right\}
$$

First we calculate the integral: 


$$
\begin{aligned}
A & =\lambda c_{d} \int_{0}^{\infty}\left(1-\exp \left(-s \eta r^{-\alpha}\right)\right) d r^{d-1} d r \\
& =\lambda c_{d} \int_{0}^{\infty}\left(1-\exp \left(-s \eta r^{-1 / \delta}\right)\right) d r \quad\left(\text { subst. } \mathrm{r} \leftarrow \mathrm{r}^{\mathrm{d}}\right) \\
& \left.=\lambda c_{d} \int_{0}^{\infty}(1-\exp (-s \eta / x)) \delta x^{\delta-1} d x \quad \text { (subst } x \leftarrow \mathrm{r}^{1 / \delta}\right)
\end{aligned}
$$

Where $\delta=d / \alpha$. To calculate this integral, we note that it is the expected value

$$
E\left[\left((X / s \eta)^{-1}\right)^{\delta}\right]
$$

Of an exponential random variable $X$ with mean 1 . Since $E\left(X^{p}\right)=\Gamma(1+p)$ by the definition of the gamma function

$$
\Gamma(p)=\int_{0}^{\infty} t^{p-1} e^{-t} d t
$$

It follows that

$$
E\left[\left((X / s \eta)^{-1}\right)^{\delta}\right]=(s \eta)^{\delta} \Gamma(1-\delta)
$$

So, with $A=\lambda c_{d}(s \eta)^{\delta} \Gamma(1-\delta)$, we obtain

$$
L_{I}(s)=\exp \left(-\lambda c_{d} E\left[\eta^{\delta}\right] \Gamma(1-\delta) s^{\delta}\right)
$$

In the case of Rayleigh fading, $E\left[\eta^{\delta}\right]=\Gamma(1+\delta)$, using the properties of the gamma function, we obtain the close-form result

$$
L_{I}(s)=\exp \left(-\lambda c_{d} s^{\delta} \frac{\pi \delta}{\sin (\pi \delta)}\right)
$$

\subsection{Interference to D2D Receiver}

We have assumed that a cell only has one cellular user, so the distribution density of cellular user is the same as the base station, here we also use $\lambda_{B}$ to present, here $\mathrm{d}=2$ and $\mathrm{c}_{\mathrm{d}}=1$. The Laplace transform of $I_{c d}$ can be obtained as [14-15]

$$
L_{\text {Icd }}(\mathrm{s})=\exp \left\{-\lambda_{B} \frac{\pi^{2} \delta}{\sin (\pi \delta)} s^{\delta} E\left[P_{C}^{\delta}\right]\right\}
$$

Where $\delta=\frac{2}{\alpha}$, because $P_{C}=h L_{C}^{\alpha \mu}$, so we have

$$
E\left[P_{C}^{\delta}\right]=E_{L_{C}}\left[h^{\delta} L_{C}^{\alpha \mu \delta}\right]=h^{\delta} \frac{R^{2 \mu}}{\mu+1}
$$

By substituting (15) into Equation (14), we get

$$
L_{\text {Icd }}(\mathrm{s})=\exp \left\{-\lambda_{B} h^{\delta} s^{\delta} \frac{\pi^{2} \delta}{\sin (\pi \delta)} \frac{R^{2 \mu}}{\mu+1}\right\}
$$

Here we let $\mu=1$, because $R=\sqrt{1 /\left(\pi \lambda_{\mathrm{B}}\right)}$, so Equation (16) will be independent of $\lambda_{\mathrm{B}}$, that is,

$$
L_{\text {Icd }}(\mathrm{s})=\exp \left\{-h^{\delta} s^{\delta} \frac{\pi \delta}{2 \sin (\pi \delta)}\right\}
$$

Since at the beginning of this article we have assumed that the distribution density of all D2D obedience Poisson process, but actually, when there is a interference restricted area in a cell, D2D users can be activated only when they located outside the restricted area, while D2D users located in the restricted area are not activated, therefore the 
activated D2D users' distribution is no longer a Poisson process, we refined it as a Poisson Hole Process, its density can be denoted as $\lambda_{D}{ }^{\prime}=\lambda_{D} \exp \left(-\lambda_{B} \pi r^{2}\right)$. So according to the lemma in [16], the Laplace transform of $I_{d d}$ can be expressed by,

$$
L_{\text {Idd }}(\mathrm{s})=\exp \left\{-h^{\delta} s^{\delta} L_{D}^{2 \mu} \frac{\pi^{2} \delta}{2 \sin (\pi \delta)} \lambda_{D} \exp \left(-\lambda_{B} \pi r^{2}\right)\right\}
$$

\subsection{Interference to BS}

In the derivation of interference to base station caused by D2D users, we make the following assumptions first:

(1) The radius of interference restricted area is not very large, because larger $r$ leads to less active DUES, thus will reduce the total spectrum efficiency.

(2) The distance between D2D pair is small, which lead to a very low transmit power, so as to reduce the interference.

(3) The number of DUES in a single cell would not be too many, because this will cause too much interference both to the CUEs and to the other DUEs.

Based on the assumptions above, we introduce in other cell's DUEs contribute little to $I_{d b}$, so the Laplace transform of $I_{d b}$ can be expressed as:

$$
L_{I_{d b}}(\mathrm{~s})=\exp \left\{-\lambda_{D} \pi E_{\eta_{v y}}\left[\left(s P_{d} \eta_{x y}\right)^{\delta} \times \gamma\left(1-\delta, \frac{s P_{d} \eta_{x y}}{R^{\alpha}}\right)-r^{2}\left(1-\exp \left(-\frac{s P_{d} \eta_{x y}}{r^{\alpha}}\right)\right)\right]\right\}
$$

Where $\gamma(a, b)=\int_{0}^{b} t^{a-1} e^{-t} d t$ is the lower incomplete gamma function, $P_{d}=h L_{D}^{\alpha \mu}$.

We can obtain the Laplace transform of $I_{c b}$ similar to (19),

$$
L_{l_{c b}}(\mathrm{~s})=\exp \left\{-\lambda_{B} \pi E_{L_{c}}\left[E_{\eta_{y y}}\left[\left(s P_{c} \eta_{x y}\right)^{\delta} \times \gamma\left(1-\delta, \frac{s P_{c} \eta_{x y}}{R^{\alpha}}\right)-R^{2}\left(1-\exp \left(-\frac{s P_{c} \eta_{x y}}{R^{\alpha}}\right)\right)\right]\right]\right\}
$$

\section{Outage Probability and Spectrum Efficiency Analysis}

\subsection{Outage Probability of Cellular Communication}

The Signal to Interference plus Noise Ratio at base station is:

$$
\operatorname{SINR}_{C}=\frac{P_{C} L_{C}^{-\alpha} \eta_{x y}}{I_{c b}+I_{d b}+n_{0}}
$$

Where $P_{C}=h L_{C}^{\alpha \mu}, n_{0}$ is the noise power density. We can obtain the occurrence probability of the cellular communication from Equation (4):

$$
P_{C}^{\prime}=P\left(\operatorname{SINR}_{C}>T\right)=P\left(\frac{P_{C} L_{C}^{-\alpha} \eta_{x y}}{I_{c b}+I_{d b}+n_{0}}>T\right)
$$

Using the fact that $\eta_{x y}$ is exponentially distributed, we can get that

$$
\begin{aligned}
P_{C}^{\prime} & =P\left(\operatorname{SINR}_{C}>T\right) \\
& =E_{L_{C}}\left[E_{I_{b}}\left[\exp \left(-T \cdot \frac{I_{b}+n_{0}}{h L_{C}^{\alpha(\mu-1)}}\right)\right]\right] \\
& =E_{L_{C}}\left[\exp \left(-T n_{0} h^{-1} L_{C}^{\alpha(1-\mu)}\right) \cdot E_{I_{b}}\left[\exp \left(-T I_{b} h^{-1} L_{C}^{\alpha(1-\mu)}\right)\right]\right]
\end{aligned}
$$

Where $I_{b}=I_{c b}+I_{d b}$, we let $\sigma=T h^{-1} L_{C}^{\alpha(1-\mu)}$, then Equation (23) can be denoted as 


$$
\begin{aligned}
P_{C}^{\prime} & =P\left(\operatorname{SINR}_{C}>T\right) \\
& =E_{L_{C}}\left[\exp \left(-\sigma n_{0}\right) \cdot E_{I_{b}}\left[\exp \left(-\sigma I_{b}\right)\right]\right] \\
& =E_{L_{C}}\left[\exp \left(-\sigma n_{0}\right) \cdot E_{I_{b}}\left[\exp \left(-\sigma\left(I_{c b}+I_{d b}\right)\right)\right]\right] \\
& =E_{L_{C}}\left[\exp \left(-\sigma n_{0}\right) \cdot L_{I_{c b}}(\sigma) \cdot L_{I_{d b}}(\sigma)\right]
\end{aligned}
$$

At last, we can get the outage probability according to $P_{C}{ }^{\prime}$

$$
P_{\text {out }, c}=1-P_{C}^{\prime}=1-E_{L_{C}}\left[\exp \left(-\sigma n_{0}\right) \cdot L_{I_{c b}}(\sigma) \cdot L_{I_{d b}}(\sigma)\right]
$$

\subsection{Outage Probability of D2D Communication}

Similar to cellular communication, we can obtain the outage probability of D2D communication. The only difference is that the distance of D2D pair is set to be constant, so the expectation based on distance can be removed, then

$$
P_{o u t, \mathrm{~d}}=1-\exp \left(-\sigma n_{0}\right) \cdot L_{I_{c d}}(\sigma) \cdot L_{I_{d d}}(\sigma)
$$

\subsection{Spectrum Efficiency Analysis}

According to Shannon theorem, we can calculate the spectral efficiency of cellular user

$$
\overline{S E_{C}}=E_{L_{C}}\left[E_{\eta}\left[\ln \left(1+S I N R_{C}\right)\right]\right]
$$

For positive random variable $\mathrm{X}$, its expectation can be expressed by [17]

$$
E(X)=\int_{0}^{\infty} P(X>t) d t
$$

So the Equation (27) can be presented as

$$
\begin{aligned}
\overline{S E_{C}} & =E_{L_{C}}\left[E_{\eta}\left[\ln \left(1+\operatorname{SINR}_{C}\right)\right]\right] \\
& =E_{L_{C}}\left[\int_{0}^{\infty} P\left(\operatorname{SINR}_{C}>e^{t}-1\right) \mathrm{dt}\right] \\
& =E_{L_{C}}\left[\int_{0}^{\infty} \frac{P\left(\operatorname{SINR} R_{C}>x\right)}{x+1} d x\right]
\end{aligned}
$$

Where $x=e^{t}-1$, by substituting (24) into Equation (29), we get

$$
\begin{aligned}
\overline{S E_{C}} & =E_{L_{C}}\left[\int_{T}^{\infty} \frac{P\left(\operatorname{SINR}_{C}>x\right)}{x+1} d x\right] \\
& =E_{L_{C}}\left[\int_{T}^{\infty} \frac{1}{x+1} \cdot \exp \left(-\xi n_{0}\right) \cdot L_{I_{c b}}(\xi) \cdot L_{I_{d b}}(\xi) d x\right]
\end{aligned}
$$

Where $\xi=x h^{-1} L_{C}^{\alpha(1-\mu)}$. In the same way, we can deduce the spectrum efficiency of D2D communication

$$
\overline{S E_{D}}=\int_{T}^{\infty} \frac{1}{x+1} \cdot \exp \left(-\xi^{\prime} n_{0}\right) \cdot L_{I_{c d}}\left(\xi^{\prime}\right) \cdot L_{I_{d d}}\left(\xi^{\prime}\right) d x
$$

Where $\xi^{\prime}=x h^{-1} L_{D}^{\alpha(1-\mu)}$.

In order to measure the performance of overall system, we introduce the concept of ASE (Area Spectral Efficiency). Specifically we compute the average spectral efficiency in units of nats/Hz, the ASE of the investigated hybrid system can be calculated as:

$$
A S E=\lambda_{C} \overline{S E_{C}}+\lambda_{D}{ }^{\prime} \overline{S E_{D}}
$$




\section{Simulation and Analysis}

In this section, we present numerical simulation of the outage probabilities and area spectral efficiency. Considering the system consist seven cells, the cell's shape is hexagonal, each cell has a central base station which use omnidirectional antenna. The same channel resource can be used by both cellular user and many D2D users, use wrap-around calculation inter-cell interference. We compare the performance in two scenarios: with restricted area and without restricted area.

\subsection{Simulation Parameters}

The simulation parameters set as shown in Table 1.

Table 1. Simulation Parameters

\begin{tabular}{|c|c|}
\hline Density of CUES $\lambda_{C}$ & $1 \times 10^{-6} \mathrm{~m}^{-2}$ \\
\hline Density of DUES $\lambda_{D}$ & $1 \times 10^{-5} \mathrm{~m}^{-2}$ \\
\hline Maximum transmitting power $P_{\max }$ & $24 \mathrm{dBm}$ \\
\hline Pass loss exponent $\alpha$ & 4 \\
\hline Rayleigh fading coefficient $\eta_{x y}$ & 0.8 \\
\hline SINR threshold T & $-10 \mathrm{~dB}$ \\
\hline system bandwidth & $1.25 \mathrm{MHZ}$ \\
\hline Noise power density $\mathrm{n}_{0}$ & $-174 \mathrm{dBm} / \mathrm{Hz}$ \\
\hline
\end{tabular}

\subsection{Outage Probability Simulation and Analysis}

Figure 4 and Figure 5 reflect that $L_{D}$ and $\lambda_{D}$ can be jointly optimized to the outage probability of both CUEs and DUEs. The red dot line indicates outage probability of D2D communication, black solid line represents outage probability of cellular communication. Figure 4 shows that: (1) when apply restricted area to base station, outage probability both D2D communication and cellular communication are reduce, especially for cellular user. This is because we assume that cellular user located in the restricted area, and D2D users that located in this restricted area are not be activated, thus reducing the interference to cellular communication that caused by the D2D communication; (2) With the increasing of $L_{D}$, outage probability both cellular communication and D2D communication are increasing. From Equation (1) we know, when the communication distance increase, the transmit power will increase, such the interference between cellular communication and D2D communication and the outage probability also increase; (3) Outage probability will decrease with the increase of restricted area, because with the increase of $r$, the activated D2D communications will reduce, this will cause the interference between D2D communications and the interference between D2D communication and cellular communication reduce correspondingly, thus reduce the communication outage probability, and the larger $r$ is, the more obvious on the improvement of the cellular communication outage probability. 


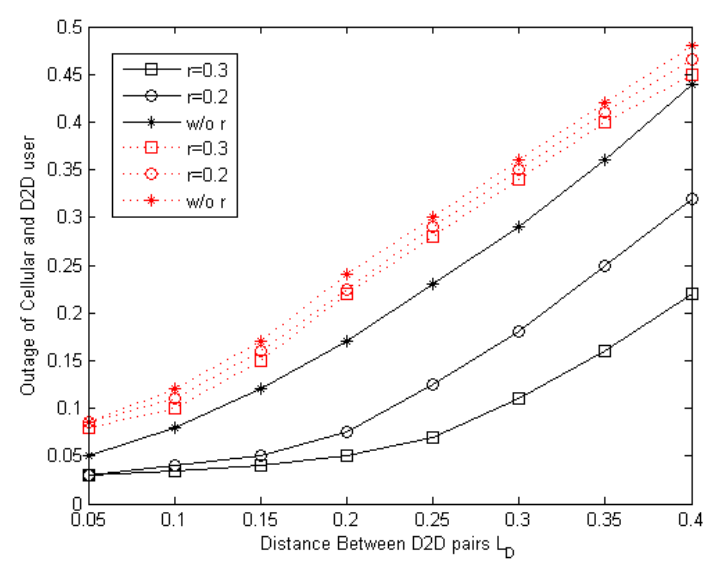

Figure 4. Outage of Cellular and D2D Users Versus $L_{D}$ Corresponds to Three Restricted Area Setting, which are Without Restricted Area, and with $\mathbf{R}=0.2,0.3$, Respectively. $\lambda_{D} / \lambda_{C}=5$

Figure 5 shows the relationship of outage probability and the density of D2D $\lambda_{D}$. As can be seen from the figure: when the distance of D2D pair is constant, outage probability will increase along with the increase of $\lambda_{D} / \lambda_{C}$, and also increase with the decrease of restricted area. But compared with Figure 4 we can find that: the increasing of interference restricted area radius have not a very obvious improvement on outage probability of D2D communication and cellular communication, for example: for cellular communication, when $\mathrm{r}=0.3$, compared with $\mathrm{r}=0.2$, the reduction of outage probability is only in $10 \%$. Therefore, when the distance of D2D pair is constant, $r$ is not the larger the better, on the contrary, small $r$ can increase the number of the activated $\mathrm{D} 2 \mathrm{D}$, thus increasing the overall system throughput and spectrum utilization. Figure 4 and Figure 5 shows that in order to improve overall system performance, select a appropriate interference region radius $r$ according to $L_{D}$ and $\lambda_{D}$ is critical.

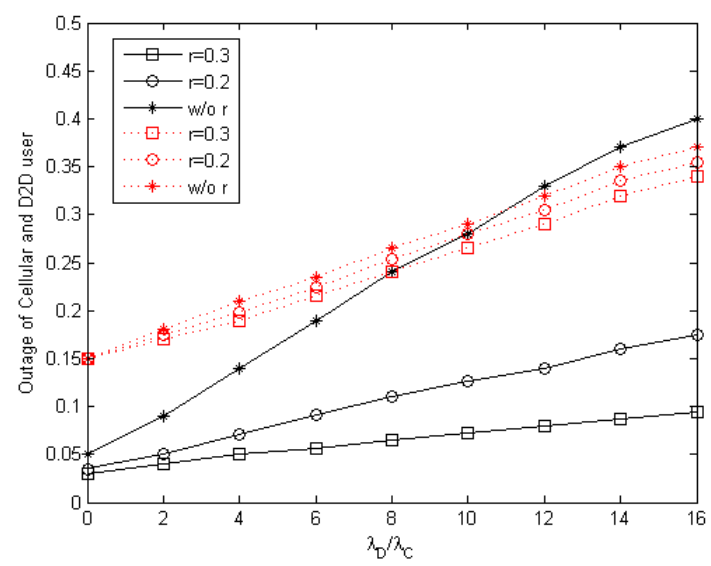

Figure 5. Outage of Cellular and D2D Users Versus $\lambda_{D}$ Corresponds to Three Restricted Area Setting, which are without Restricted Area, and with R=0.2, 0.3, Respectively. $L_{D}=0.5$ 


\subsection{Area Spectral Efficiency Simulation and Analysis}

Figure 6 and Figure 7 show the area spectral efficiency versus D2D communication distance $L_{D}$, the density $\lambda_{D}$ variation of D2D. Figure 6 indicates that for the different interference restricted area radius $r$, the spectral efficiency will become larger as the distance of D2D communication decrease, and when $L_{D}<0.1$, the scheme without restricted area actually leads to better area spectral efficiency, this is because when $L_{D}$ is very small, according to the dynamic power control algorithm mentioned before, transmit power of D2D will be smaller accordingly, so the interference between communication will be smaller. If increase the interference restricted area, it will make the activated D2D fewer, so that the spectral efficiency of the system will become smaller. Obviously, there is no necessary to increase the interference restricted area. When $L_{D}>0.1$, the area spectral efficiency with interference restricted area is higher than those without, but the improvement is not obvious.

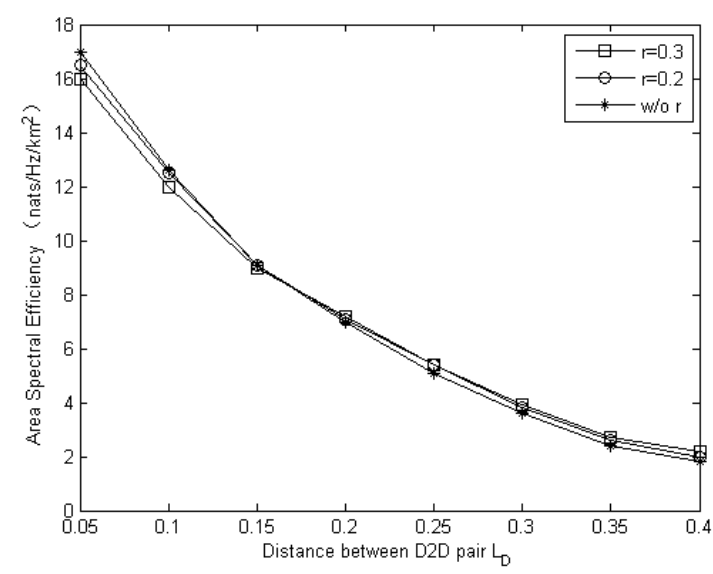

Figure 6. Area Spectral Efficiency Versus $L_{D}$ Corresponds to Three Restricted Areas, which are without Restricted Area, and With R=0.2, 0.3, Respectively. $\lambda_{D} / \lambda_{C}=5$

Figure 7 illustrates the impact of D2D density $\lambda_{D}$ on the area spectral efficiency. When $L_{D}=0.08$, area spectral efficiency will increase as the density of D2D users increase, and we can also conclude from the figure, the interference restricted area does not increase the spectral efficiency of the system. This is also verified the reaction in Figure 6, when the distance between the D2D pair is small, the set of interference restricted area is not necessary, but with the $L_{D}$ increasing, the transmit power of D2D becomes large, the interference between communication system will become strong corresponding, then the set of interference restricted area will reduce interference, improve system performance. For example, $L_{D}=0.3$, when $\lambda_{D} / \lambda_{C}>6$, due to the interference between the communication become more and more strong, the spectrum efficiency of the system will reduce, but the area spectral efficiency with a interference restricted area is higher than that without interference restricted area. In general, when $L_{D}$ is small, the set of interference restricted area is not necessary; when $L_{D}$ is large, interference restricted area can reduce interference and improve system area spectral efficiency obviously. 


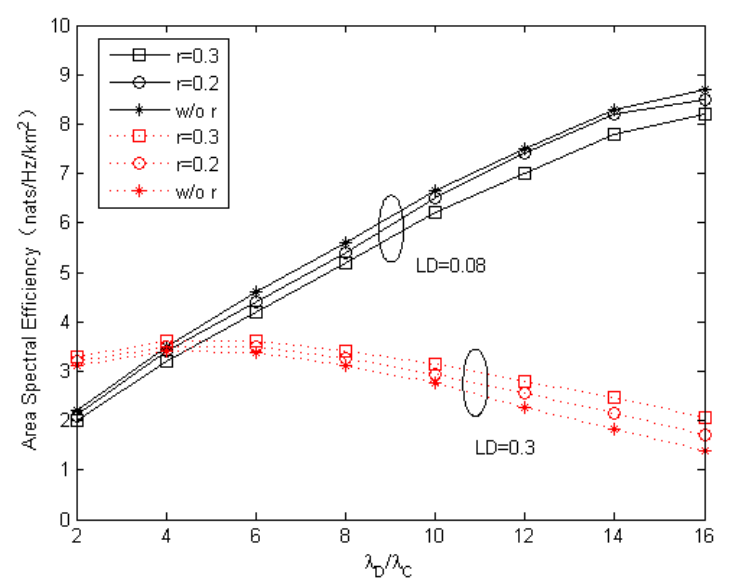

Figure 7. Area Spectral Efficiency Versus $\lambda_{D}$ Correspond to Three Restricted Areas, which are without Restricted Area, and with $\mathrm{R}=0.2,0.3$,
Respectively

\section{Conclusion}

This paper from the view of further enhance system spectral efficiency and reduce interference between the communication, proposed a D2D dynamic power control algorithm based on interference restricted area in multi-cell and multi D2D communications scenario. According to channel gain and the communication distance dynamically adjust the transmit power of each transmitter, and combine interference restricted area to reduce interference between communications. Simulation results show that the proposed algorithm can reduce the outage probability of communication, improve system spectral efficiency while allowing the system have more D2D pairs, thereby increasing the overall throughput of the system.

\section{Acknowledgement}

This work was supported by the Open Research Fund of National Mobile Communications Research Laboratory, Southeast University (No.2014D13) and Gansu Province Natural Science Foundation (No.1310RJZA003).

\section{References}

[1] S. Land and J. Shen "Evolved Network Planning and Optimization for UMTS and LTE", Auerbach Publications, CRC Press, (2010).

[2] K. Doppler, M. P. Rinne, C. Wijting, C. B. Ribeiro and K. Hugl, "Device-to-Device Communication as an Underlay to LTE-Advanced Networks", IEEE Communications Magazine, vol. 47, no. 12, pp. 42-49, (2009).

[3] A. Osseiran, V. Braun and T. Hidekazu, "The foundation of the mobile and wireless communications system for 2020 and beyond challenges, enablers and technology solutions", In 2013 IEEE 77th Vehicular Technology Conference(VTC Spring), Dresden, Germany, (2013), pp. 1-5.

[4] C. H. Yu, O. Tirkkonen and K. Doppler, "Power optimization of device-to-device communication underlaying cellular communication", ICC'09 IEEE International Conference on Communications. IEEE, (2009), pp. 1-5.

[5] G. Fodor and N. Reider, "A distributed power control scheme for cellular network assisted D2D communication", IEEE Global Telecommunications Conference, (2011), pp. 1-6.

[6] N. Lee, L. Xingqin and W. Robert, "Power Control for D2D Underlaid Cellular Network: Modeling, Algorithms, and Analysis", IEEE Trans. Journal Ares in Communications, vol. 33, no. 1, (2015), pp. 113 . 
[7] J. Gu, S. J. Bae, B. G. Choi and M. Y. Chung, "Dynamic power control Mechanism for interference coordination of device-to-device communication in cellular networks", in Proceeding 3rd Int. Conference Ubiquitous Future Network, (2011), pp. 71-75.

[8] H. Min, J. Lee, S. Park and D. Hong, "Capacity enhancement using an interference limited area for device-to-device uplink underlaying cellular networks", IEEE Transactions in Wireless Communication, DOI:10.1109/TWC.(2011).100611.101684, vol. 10, no. 12, (2011), pp. 3995-4000.

[9] X. Xiao, T. Xiaoming and L. Jianhua, "A Qos-aware power optimization scheme in OFDMA system with integrated device-to-device (D2D) communication", IEEE Vehicular Technology Conference, (2011), pp. 1-5.

[10] M. G. da S. Rego, T. F. Maciel, H. de H. M .Barros and G. Fodor, "Performance analysis of power control for device-to-device communication in cellular MIMO systems", in International Symposium on Wireless Communication Systems, (2012), pp. 336-340.

[11] P. Janis, V. Koivunen, C. Ribeiro, J. Korhonen, K. Doppler and K. Hugl, "Interference-aware resource allocation for device-to-device radio underlaying cellular networks", in Proc. IEEE 69th Vehicular Technology Conference, Barcelona, Spain, (2009), pp. 1-5.

[12] E. Biglieri, G. Caire and G. Taricco, "Limiting performance of block-fading channels with antennas", IEEE Trans on Information Theory, vol. 47, no. 4, (2001), pp. 1273-1289.

[13] H. Wang and X. Chu, "Distance-constrained resource-sharing criteria for device-to-device communications underlaying cellular networks", Electronic Letters, DOI:10.1049/el.(2012).0415, vol. 48, no. 9, (2012), pp. 528-530.

[14] M. Haenggi and P. K. Ganti, "Interference in Large Wireless Networks", Now Publishers Inc.: BostonDelft, (2009).

[15] X. Zhang and M. Haenggi, "Random power control in poison networks", IEEE Transactions on Communications, DOI: 10.1109/TCOMM. (2012).071312.110730, vol. 60, no. 9, (2012), pp. 26022611.

[16] C. H. Lee and M. Haenggi, "Interference and outage in Poisson cognitive networks", IEEE Transactions on Wireless Communications, vol. 11, no. 4, (2012), pp. 1392-1401.

[17] R. K. Ganti, F. Baccelli and J. G. Andrew, “A new way of computing rate in cellular networks”, In 2011 IEEE International Conference on Communications (ICC), Kyoto, Japan, DOI: 10.1109/icc.(2011).5962727, (2011), pp.1-5.

[18] Qualcomm Inc. CDMA Uplink Power Control Methodology for SEAMCAT(Voice Only)[EB/OL]. (2003-08-12). http://www.ero.dk/F9669EF2-85DD-4F0C-92ED-F1A53D5DF907?frames=no\&.

[19] X. Junfeng, Z. Shihong and Q. Jing, "Across-layer adaptive transmission scheme combined with SRARQ over correlated fading channels", Computers and Electrical Engineering, vol. 34, no. 4, (2008), pp. 324-337.

[20] L. Qingwen, Z. Shengli and G. B. Giannakis, "Cross-layer combining of adaptive modulation and coding with truncated ARQ over wireless links", IEEE Trans on Wireless Communication, vol. 3, no. 5, (2004), pp. 1746-1755.

[21] E. Yaacoub, "On the use of device-to-device communications for QoS and data rate enhancement in LTE public safety networks", presented at the IEEE WCNC-Workshop Device-to-Device Public Safety Communications, Istanbul, Turkey, (2014).
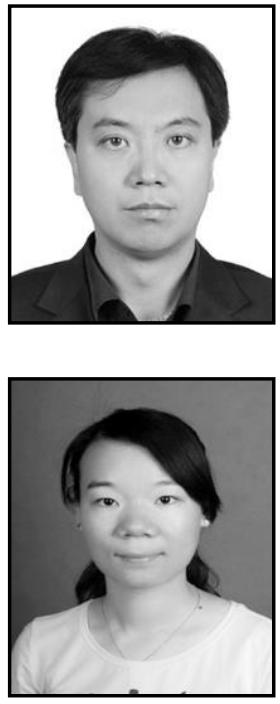

\section{Authors}

Jianbin Xue, works as a professor in School of Computer and Communication, LanZhou University of Technology. He received Doctor's degree of Control Theory and Control Engineering in 2009 from LanZhou University of Technology. His current research interests are Wireless communication theory and technology; Media information processing; System modeling and simulation.

Supan Wei, is pursuing Master's degree in Communication and Information System in LanZhou University of Technology. She received bachelor's degree from LuoYang University of Technology in 2012. Research Interests: Device-to-Device Communication; Wireless communication theory and technology. 


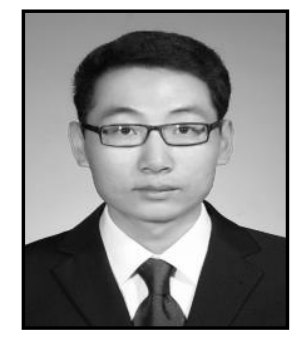

Yiming Chen, is pursuing Master's degree in Internet of Things in LanZhou University of Technology. He received bachelor's degree from Nanchang Institute of Technology in 2013. Research Interests: Underwater sensor; IoT. 
International Journal of Multimedia and Ubiquitous Engineering Vol.11, No.5 (2016) 